\title{
Coronary Perfusion Pressure Response to High-Dose Intraosseous versus Standard-Dose Intravenous Epinephrine Administration after Prolonged Cardiac Arrest
}

\author{
Timothy J. Mader*, Ryan A. Coute, Adam R. Kellogg, Joshua L. Harris \\ Department of Emergency Medicine at Baystate Medical Center, Tufts University School of Medicine, \\ Springfield, MA, USA \\ Email: ${ }^{*}$ timothy.mader@baystatehealth.org
}

Received 6 January 2014; revised 4 February 2014; accepted 3 March 2014

Copyright (C) 2014 by authors and Scientific Research Publishing Inc. This work is licensed under the Creative Commons Attribution International License (CC BY). http://creativecommons.org/licenses/by/4.0/ (c) (i) Open Access

\begin{abstract}
Background: This study was done to compare coronary perfusion pressure (CPP) prior to the first rescue shock (RS) among a group of animals that received intraosseous (IO) epinephrine $0.1 \mathrm{mg}$ / kg (high-dose epinephrine [HDE]) with a group that received intravenous (IV) epinephrine 0.01 $\mathrm{mg} / \mathrm{kg}$ (standard-dose epinephrine [SDE]) during cardiac arrest resuscitation using a swine model of prolonged out-of-hospital ventricular fibrillation (VF) cardiac arrest. Methods: This was a secondary analysis of prospectively collected data from two IACUC approved protocols. Seventy-nine Yorkshire swine (25 - $35 \mathrm{~kg}$ ) were surgically instrumented under anesthesia and VF was electrically induced. After 10 minutes of untreated VF in the IO study $(n=26)$ and 12 minutes of untreated VF in the IV study ( $n=53)$, resuscitation commenced with closed chest compressions (CCC). A single dose of epinephrine (HDE IO or SDE IV, respectively) was given and flushed with saline. The CCC and RS attempts were standardized for all animals. The CPP was defined as aortic diastolic pressure minus right atrial diastolic pressure measured 2.5 minutes after medication delivery. Descriptive statistics were used to analyze the data. Results: Baseline group characteristics were mathematically the same. Just prior to the first RS, HDE IO resulted in a mean CPP of $33.2 \mathrm{mmHg}$ (95\%CI: 26.6, 39.9), while SDE IV resulted in a mean CPP of $25.0 \mathrm{mmHg}(95 \% \mathrm{CI}$ : 20.5, 29.4). Conclusion: This observation study reaffirms the assertion that HDE IO may be required to generate CPP values similar to SDE IV during resuscitation of prolonged VF.
\end{abstract}

\footnotetext{
*Corresponding author.
} 


\section{Keywords}

\section{Cardiac Arrest Resuscitation; Coronary Perfusion Pressure; Intraosseous; Epinephrine}

\section{Introduction}

While provision of vasopressors during attempted resuscitation of out-of-hospital cardiac arrest (OHCA) victims has never been shown to improve neurologically intact survival, its short-term benefits are clear [1]. Despite evidence that the intraosseous (IO) dose of epinephrine should be substantially higher than that given intravenous (IV) to achieve pharmacokinetic and hemodynamic equivalency [2], current guidelines still recommend a dose of $0.01 \mathrm{mg} / \mathrm{kg}$ regardless of delivery route (IO or IV) [3]. This study was done to compare CPP prior to the first attempted defibrillation between a group of animals that received epinephrine $0.1 \mathrm{mg} / \mathrm{kg}$ (HDE) IO with a group that received epinephrine $0.01 \mathrm{mg} / \mathrm{kg}$ (SDE) IV during cardiac arrest resuscitation using a swine model of prolonged out-of-hospital ventricular fibrillation (VF) cardiac arrest.

\section{Methods}

This was a secondary analysis of prospectively collected data from 2 previously completed studies. Both studies were approved by the Institutional Animal Care and Utilization Committee (IACUC), and conducted in our USDA-certified laboratory. We used 79 locally obtained domestic Yorkshire swine, weighing approximately 25 $35 \mathrm{~kg}$. Animals were delivered and acclimated in advance of use. Our standard animal preparation and resuscitation procedures have been previously described in detail [4] [5].

Briefly, at the time of surgery, we sedated the animal with intramuscular TKX [telazol (5 mg/kg), ketamine ( $2.5 \mathrm{mg} / \mathrm{kg})$, and xylazine $(2.5 \mathrm{mg} / \mathrm{kg})]$. We provided inhaled isoflurane to facilitate advanced airway placement with a size-appropriate cuffed endotracheal tube and obtain intravenous access. We established a surgical plane of anesthesia using a rapid IV injection of propofol $(2 \mathrm{mg} / \mathrm{kg})$ and maintained this with a continuous infusion (80 $\mathrm{mics} / \mathrm{kg} / \mathrm{min}$ ), titrated to effect. During preparation, we ventilated the animals with room air, using a volume-cycled ventilator, and adjusted the tidal volume and ventilation rate to maintain eucapnea $\left(\mathrm{EtCO}_{2}=38\right.$ - 42 torr). We secured surface electrodes configured to correspond to a standard lead II electrocardiogram (ECG).

After the airway was secured and a surgical depth of anesthesia was established, we induced neuromuscular paralysis with pancuronium (4 mg initial bolus IV). We then placed an arterial introducer (8.5 Fr) into the femoral artery and a venous introducer (8.5 Fr) into the femoral vein under direct visualization. We inserted micro-manometer tipped pressure catheters (Mikro-Tip, Millar Instruments, Houston, TX) through the introducers and advance them into the ascending aorta and right atrium, respectively. Proper positioning of the catheters was confirmed by interpretation of the pressure tracings. All central vascular access ports were connected to a pressurized liter bag of normal saline containing heparin.

We analyzed an ABG (I-Stat portable Clinical Analyzer, Heska Corp, Waukesha, WI) as soon as access was established and just before the induction of VF. The ECG tracing and all pressure data were continuously monitored and digitally recorded via a commercially available software package (LabChart, v.7.2.3, AD Instruments, Colorado Springs, CO). Finally, we made a record of all baseline measures for group comparisons.

Immediately before induction of VF, a 2-mg bolus of pancuronium was given, the propofol infusion was discontinued, and the ventilator was disconnected. We induced VF by delivering a 3-second, $60-\mathrm{Hz}, 100-\mathrm{mA}$ transthoracic alternating current using a PowerStat variable transformer. The occurrence of VF was confirmed by the abrupt loss of aortic pressure waves and the characteristic ECG appearance.

After 10 minutes of untreated VF in the IO study $(n=26)$ and 12 minutes of untreated VF in the IV study $(n=$ 53), when cardiac arrest for all animals had crossed into the metabolic phase [6], resuscitation commenced with closed chest compressions (CCC). We used an oxygen-powered mechanical resuscitation device (Life-Stat ${ }^{\mathrm{TM}}$ Mechanical CPR System, Michigan Instruments, Grand Rapids, MI) that provides standardized chest compressions in the anterior-posterior direction at a rate of 100 per minute with complete chest recoil. To minimize chest wall trauma, the compression depth was gradually adjusted over 20 seconds to generate peak aortic pressure spikes of 40 - $50 \mathrm{mmHg}$ (1.25 - 2 inches in depth).

In the IO group, a pediatric needle (EZ-IO® Intraosseous system, VidaCare Corporation) was inserted in real 
time for vascular access for epinephrine delivery. Correct placement of the IO needle was confirmed by stability of the needle, marrow aspiration upon insertion, and direct examination on necropsy at the conclusion of the experiment. In the IV group, the vascular access established during preparation phase of the experiment (described above) via the femoral vein was used for epinephrine delivery. After 30 seconds of initial chest compressions, a single dose of epinephrine (HDE IO or SDE IV, respectively) was given followed by a large volume saline flush. An additional 2.5 minutes of compressions were provided after injection to circulate the medication before the first rescue shock (RS) was delivered. All defibrillation attempts were delivered at a fixed dose of energy $(120 \mathrm{~J})$ with a proprietary Rectilinear Biphasic ${ }^{\mathrm{TM}}$ defibrillation waveform (E Series ${ }^{\mathrm{TM}}$, Zoll Medical Corp., Chelmsford, MA) through adult-sized defibrillator pads (Adult Plus multifunction electrode pads, Philips Healthcare, Andover, MA).

The CPP was defined as aortic diastolic pressure minus right atrial diastolic pressure, and the values were extracted immediately following the last compression before defibrillation for the first RS in each animal (Figure 1). The data was analyzed descriptively using a commercially available software package (Stata/SE v.10.0 for Macintosh, College Station, TX).

\section{Results}

Baseline group characteristics prior to VF induction were mathematically the same (Table 1). To avoid the possibility of the groups in the parent studies being unbalanced on animal sex, only females were used in both. The pre-anesthesia time refers to the period between TKX injection and the propofol bolus. The anesthesia time refers to the period between propofol bolus and VF induction. After 3 minutes of resuscitation, HDE IO produced a mean CPP of $33.2 \mathrm{mmHg}$ (95\%CI: 26.6, 39.9), while SDE IV resulted in a mean CPP of $25.0 \mathrm{mmHg}$ (95\%CI: 20.5, 29.4) just prior to $\mathrm{RS}_{1}$ (Figure 2). Despite a 10-fold greater dose delivered to the IO group, the CPP was only $33 \%$ higher than the IV group. This difference was not statistically significant.

\section{Discussion}

The generation of a CPP value adequate for myocardial perfusion (typically quoted as 15 - $25 \mathrm{mmHg}$ ) after prolonged VF cardiac arrest is key to defibrillation success, ROSC, and short-term survival [7]-[21]. While high quality continuous chest compressions during cardiopulmonary resuscitation can provide cerebral and myocardial blood flow, a threshold CPP sufficient for defibrillation success, ROSC, and short-term survival in the metabolic phase of cardiac arrest is unlikely to be achieved without vasopressors. The IO route has been used for vascular access and medication deliver in pediatric emergency care for over 30 years, while gaining acceptance for establishing vascular access and giving resuscitation medications in adults only more recently. With the rap-

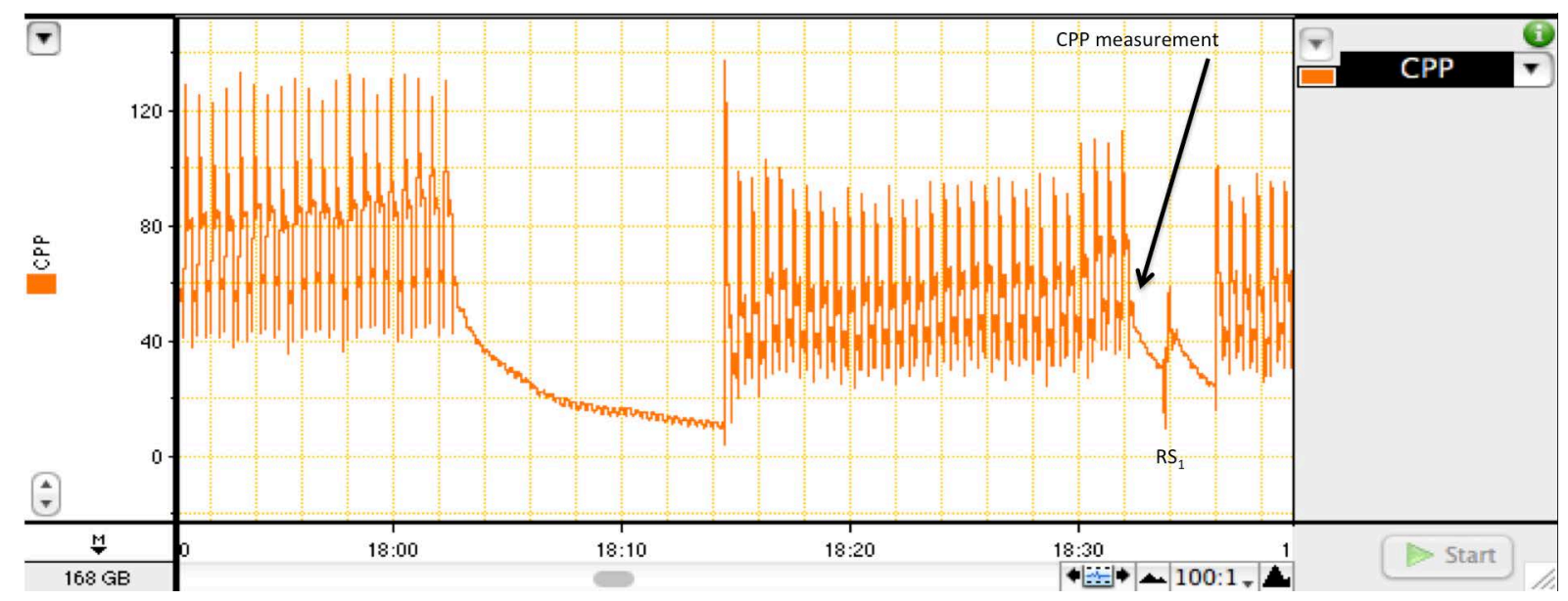

Figure 1. The coronary perfusion pressure tracing from one of the animals just prior to the first rescue shock $\left(\mathrm{RS}_{1}\right)$, demonstrating the point from which the CPP measurements were taken. The chest compression pause from 18:03 to 18:14 was by design to simulate a 10-second clinical rhythm check, during which time we recorded an artifact-free segment of the ECG channel for quantitative waveform analysis. 


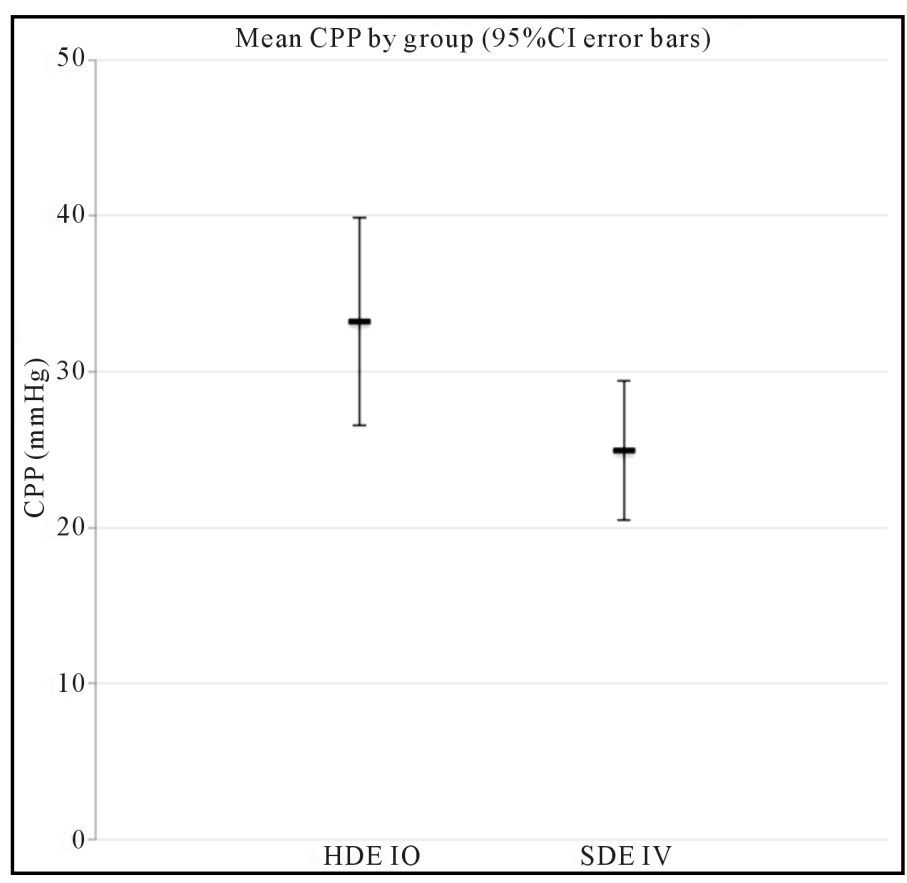

Figure 2. Graphic representation of our results. After 3 minutes of resuscitation, HDE IO produced a mean CPP of $33.2 \mathrm{mmHg}$ (95\%CI: 26.6, 39.9), while SDE IV resulted in a mean CPP of $25.0 \mathrm{mmHg}$ (95\%CI: 20.5, 29.4) just prior to $\mathrm{RS}_{1}$. Despite a 10 -fold greater dose delivered to the IO group, the CPP was only $33 \%$ higher than the IV group. This difference was not statistically significant.

Table 1. Baseline characteristics

\begin{tabular}{ccc}
\hline & HDE IO (n=26) & SDE IV (n = 53) \\
\hline Weight (kg) & $31.0(3.3)$ & $32.4(2.7)$ \\
Pre-anesthesia time (minutes) & $18.2(7.0)$ & $20.0(4.1)$ \\
Anesthesia time (minutes) & $26.7(5.2)$ & $41.2(2.8)$ \\
End tidal carbon dioxide (mmHg) & $40.6(2.0)$ & $37.6(0.9)$ \\
Temperature ( $\left.{ }^{\circ} \mathrm{C}\right)$ & $37.7(0.8)$ & $108.0(13.4)$ \\
Mean arterial pressure (mmHg) & $94.8(15.5)$ & $122.6(18.9)$ \\
Heart rate (bpm) & $115.8(17.7)$ & $92.7(23.2)$ \\
Blood glucose (mg/dL) & $100.3(44.2)$ & $139.5(1.7)$ \\
Sodium (mEq/L) & $139.9(1.9)$ & $4.0(0.4)$ \\
Potassium (mEq/L) & $4.0(0.3)$ & $1.38(0.06)$ \\
Ionized calcium (mmol/L) & $1.36(0.07)$ & $27.7(2.4)$ \\
Hematocrit (\%) & $26.7(4.1)$ & $7.49(0.04)$ \\
pH (units) & $7.51(0.04)$ & $40.1(3.3)$ \\
Partial pressure of carbon dioxide (mmHg) & $37.3(3.0)$ & $88.5(10.1)$ \\
Partial pressure of oxygen (mmHg) & $89.5(12.2)$ & All values are group means (Standard Deviation).
\end{tabular}

id proliferation of IO use in adult cardiac arrest resuscitation, it is important to determine if vasopressor dosages need to be adjusted for the route of administration. 
Current guidelines for epinephrine administration during cardiac arrest resuscitation recommend a dose of $0.01 \mathrm{mg} / \mathrm{kg}$ regardless of delivery route (IO or IV) [3], despite evidence that the IO dose should be substantially higher to achieve pharmacokinetic and hemodynamic equivalency [2]. Two decades ago, Spivey et al., using domestic swine (12 - $15 \mathrm{~kg}$ ) subjected to 5 minutes of untreated VF and resuscitated using 60 chest compressions per minute with cycled ventilation at a ratio of 5:1, determined that SDE IO did not produce an appreciable change in plasma epinephrine levels or mean arterial pressure while HDE IO resulted in a dramatic increase in plasma epinephrine levels and mean arterial pressure. There has been a paucity of literature on this topic since this research was first published, this issue is still unresolved, and the guidelines have yet to be modified.

This study, done to compare CPP prior to the first attempted defibrillation between a group of animals that received HDE IO with a group that received SDE IV during cardiac arrest resuscitation using a swine model of prolonged out-of-hospital VF cardiac arrest, found that the hemodynamic effects were equivalent, reaffirming the assertion that the IO dose of epinephrine for cardiac arrest resuscitation should be substantially higher than that given IV to achieve pharmacokinetic and hemodynamic equivalency.

We must acknowledge that vascular access established during preparation phase of the experiment via the femoral vein was used for SDE delivery rather than a peripheral IV, which may have skewed the data in favor of the SDE IV group. Use of an auricular vein in this model might be a more clinically relevant comparator, though from our previous work we note that HDE through the femoral vein resulted in a peak CPP of $34.8 \mathrm{mmHg}$ (95\%CI: 25.2, 44.5), values quite similar to the HDE IO group in this study [5]. This does however raise concerns regarding the appropriateness of the SDE IV dosage recommendation in the metabolic phase of cardiac arrest, and its ability when delivered peripherally to generate adequate CPP sufficient for defibrillation success, ROSC, and short-term survival in a substantial number of OHCA victims.

\section{Study Limitations}

There are several important limitations to this study. First, these data were obtained under experimental conditions using healthy swine and electrically induced VF cardiac arrest. How well these findings translate to human OHCA is uncertain. Second, the duration of untreated VF in the two studies was not the same, however, resuscitation was not initiated in any animal until cardiac arrest had reached the metabolic phase of VF making the groups sufficiently comparable to be analyzed together. As such, these results cannot be extrapolated to the electrical or circulatory phase of cardiac arrest. Third, plasma catecholamine levels were not measured over time, so it is possible that the physiologic response observed was due to differences in drug distribution and timing of peak levels in the central circulation rather than the dose delivered. Fourth, the studies were not done concurrently and may have been subject to unmeasured confounders that may have affected the data. There were, however, no significant differences in the protocols or changes in laboratory conditions between the conduct of the two experiments. Finally, we did not directly compare HDE IO with SDE IO and therefore cannot comment on whether or not similar CPP values might have been observed with SDE IO. We encourage further research along this line of investigation to address these limitations.

\section{Conclusion}

This observation study reaffirms the assertion that HDE given via the IO route may be required to generate CPP values similar to SDE delivered IV during resuscitation of prolonged out-of-hospital VF cardiac arrest. A prospective randomized comparison of HDE IO versus SDE IO in the metabolic phase of VF measuring plasma catecholamine levels is needed to test this hypothesis and to determine the impact on ROSC and short-term survival.

\section{Acknowledgements}

Project grants through the Baystate Health Incubator Fund Program supported the investigator-initiated studies from which these data were extracted. The authors appreciate the contribution of VidaCare Corporation for donating the EZ-IO ${ }^{\circledR}$ Intraosseous system and the related supplies used in the HDE IO parent study-no other consideration was provided.

\section{Contributors}

TJM designed the study, performed data collection, performed the data analysis, and wrote the manuscript. RAC 
performed data collection and assisted with writing the manuscript. ARK was involved with data collection and edited the manuscript. TJM is responsible for this work as guarantor.

\section{Prior Presentation}

American Heart Association Resuscitation Science Symposium, November 2012. Los Angeles, CA. National Association of EMS Physicians, January 2013. Bonita Springs, FL.

\section{References}

[1] Callaway, C.W. (2013) Epinephrine for Cardiac Arrest. Current Opinion in Cardiology, 28, 36-42. http://dx.doi.org/10.1097/HCO.0b013e32835b0979

[2] Spivey, W.H., Crespo, S.G., Fuhs, L.R. and Schoffstall, J.M. (1992) Plasma Catecholamine Levels after Intraosseous Epinephrine Administration in a Cardiac Arrest Model. Annals of Emergency Medicine, 21, 127-131. http://dx.doi.org/10.1016/S0196-0644(05)80145-3

[3] Neumar, R.W., Otto, C.W., Link, M.S., et al. (2010) Part 8: Adult Advanced Cardiovascular Life Support: 2010 American Heart Association Guidelines for Cardiopulmonary Resuscitation and Emergency Cardiovascular Care. Circulation, 122, S729-S767. http://dx.doi.org/10.1161/CIRCULATIONAHA.110.970988

[4] Mader, T.J., Kellogg, A.R., Smith, J., et al. (2008) A Blinded, Randomized Controlled Evaluation of an Impedance Threshold Device During Cardiopulmonary Resuscitation in Swine. Resuscitation, 77, 387-394. http://dx.doi.org/10.1016/j.resuscitation.2008.01.007

[5] Mader, T.J., Kellogg, A.R., Walterscheid, J.K., Lodding, C.C. and Sherman, L.D. (2010) A Randomized Comparison of Cardiocerebral and Cardiopulmonary Resuscitation Using a Swine Model of Prolonged Ventricular Fibrillation. Resuscitation, 81, 596-602. http://dx.doi.org/10.1016/j.resuscitation.2010.01.013

[6] Weisfeldt, M.L. and Becker, L.B. (2002) Resuscitation after Cardiac Arrest: A 3-Phase Time-Sensitive Model. JAMA: The Journal of the American Medical Association, 288, 3035-3038. http://dx.doi.org/10.1001/jama.288.23.3035

[7] Redding, J.S. and Pearson, J.W. (1963) Evaluation of Drugs for Cardiac Resuscitation. Anesthesiology, 24, $203-207$. http://dx.doi.org/10.1097/00000542-196303000-00008

[8] Babbs, C.F. (1980) New versus Old Theories of Blood Flow during CPR. Critical Care Medicine, 8, 191-195. http://dx.doi.org/10.1097/00003246-198003000-00026

[9] Ditchey, R.V., Winkler, J.V. and Rhodes, C.A. (1982) Relative Lack of Coronary Blood Flow during Closed-Chest Resuscitation in Dogs. Circulation, 66, 297-302. http://dx.doi.org/10.1161/01.CIR.66.2.297

[10] Sanders, A.B., Ewy, G.A., Alferness, C.A., Taft, T. and Zimmerman, M. (1982) Failure of One Method of Simultaneous Chest Compression, Ventilation, and Abdominal Binding during CPR. Critical Care Medicine, 10, 509-513. http://dx.doi.org/10.1097/00003246-198208000-00005

[11] Michael, J.R., Guerci, A.D., Koehler, R.C., et al. (1984) Mechanisms by which Epinephrine Augments Cerebral and Myocardial Perfusion during Cardiopulmonary Resuscitation in Dogs. Circulation, 69, 822-835. http://dx.doi.org/10.1161/01.CIR.69.4.822

[12] Niemann, J.T., Rosborough, J.P., Niskanen, R.A., Alferness, C. and Criley, J.M. (1985) Mechanical “Cough” Cardiopulmonary Resuscitation during Cardiac Arrest in dogs. The American Journal of Cardiology, 55, 199-204. http://dx.doi.org/10.1016/0002-9149(85)90328-5

[13] Niemann, J.T., Criley, J.M., Rosborough, J.P., Niskanen, R.A. and Alferness, C. (1985) Predictive Indices of Successful Cardiac Resuscitation after Prolonged Arrest and Experimental Cardiopulmonary Resuscitation. Annals of Emergency Medicine, 14, 521-528. http://dx.doi.org/10.1016/S0196-0644(85)80774-5

[14] Sanders, A.B., Kern, K.B., Atlas, M., Bragg, S. and Ewy, G.A. (1985) Importance of the Duration of Inadequate Coronary Perfusion Pressure on Resuscitation from Cardiac Arrest. Journal of the American College of Cardiology, 6, 113-118. http://dx.doi.org/10.1016/S0735-1097(85)80261-8

[15] Kern, K.B., Ewy, G.A., Voorhees, W.D., Babbs, C.F. and Tacker, W.A. (1988) Myocardial Perfusion Pressure: A Predictor of 24-Hour Survival during Prolonged Cardiac Arrest in Dogs. Resuscitation, 16, 241-250. http://dx.doi.org/10.1016/0300-9572(88)90111-6

[16] Paradis, N.A., Martin, G.B., Rivers, E.P., et al. (1990) Coronary Perfusion Pressure and the Return of Spontaneous Circulation in Human Cardiopulmonary Resuscitation. JAMA: The Journal of the American Medical Association, 263, 1106-1113. http://dx.doi.org/10.1001/jama.1990.03440080084029

[17] Cobb, L.A., Fahrenbruch, C.E., Walsh, T.R., et al. (1999) Influence of Cardiopulmonary Resuscitation Prior to Defibrillation in Patients with Out-of-Hospital Ventricular Fibrillation. JAMA: The Journal of the American Medical Asso- 
ciation, 281, 1182-1188. http://dx.doi.org/10.1001/jama.281.13.1182

[18] Weisfeldt, M.L. and Becker, L.B. (2002) Resuscitation after Cardiac Arrest: A 3-Phase Time-Sensitive Model. JAMA: The Journal of the American Medical Association, 288, 3035-3038. http://dx.doi.org/10.1001/jama.288.23.3035

[19] Wik, L., Hansen, T.B., Fylling, F., et al. (2003) Delaying Defibrillation to Give Basic Cardiopulmonary Resuscitation to Patients with Out-of-Hospital Ventricular Fibrillation: A Randomized Trial. JAMA: The Journal of the American Medical Association, 289, 1389-1395. http://dx.doi.org/10.1001/jama.289.11.1389

[20] Berg, R.A., Hilwig, R.W., Ewy, G.A. and Kern, K.B. (2004) Precountershock Cardiopulmonary Resuscitation Improves Initial Response to Defibrillation from Prolonged Ventricular Fibrillation: A Randomized, Controlled Swine Study. Critical Care Medicine, 32, 1352-1357. http://dx.doi.org/10.1097/01.CCM.0000127780.01362.E5

[21] Reynolds, J.C., Salcido, D.D. and Menegazzi, J.J. (2010) Coronary Perfusion Pressure and Return of Spontaneous Circulation after Prolonged Cardiac Arrest. Prehospital Emergency Care, 14, 78-84. http://dx.doi.org/10.3109/10903120903349796 